\title{
Universiteit
}

Leiden

The Netherlands

\section{Magnetic coupling in superconducting spin valves with strong}

ferromagnets

Flokstra, M.G.; Knaap, J.M. van der; Aarts, J.

\section{Citation}

Flokstra, M. G., Knaap, J. M. van der, \& Aarts, J. (2010). Magnetic coupling in superconducting spin valves with strong ferromagnets. Physical Review B, 82, 184523. doi:10.1103/PhysRevB.82.184523

Version: $\quad$ Not Applicable (or Unknown)

License: $\quad$ Leiden University Non-exclusive license

Downloaded from: https://hdl.handle.net/1887/44543

Note: To cite this publication please use the final published version (if applicable). 


\title{
Magnetic coupling in superconducting spin valves with strong ferromagnets
}

\author{
M. Flokstra, J. M. van der Knaap, and J. Aarts \\ Kamerlingh Onnes Laboratory, Leiden Institute of Physics, P.O. Box 9504, 2300 RA Leiden, The Netherlands
}

(Received 9 August 2010; published 15 November 2010)

\begin{abstract}
We investigate the magnetotransport behavior of ferromagnet $(\mathrm{F}) /$ superconductor/ferromagnet trilayers made of ferromagnetic $\mathrm{Ni}_{80} \mathrm{Fe}_{20}$ (Permalloy, Py) and superconducting $\mathrm{Nb}$ for temperatures both above and below the superconducting transition temperature $T_{c}$. In such devices, and for weak ferromagnets, $T_{c}$ depends on the relative magnetization directions of the two $\mathrm{F}$ layers in such a way that $T_{c}^{\mathrm{P}}$ of the parallel (P) alignment is lower than $T_{c}^{\mathrm{AP}}$ of the antiparallel (AP) alignment (the so-called superconducting spin-valve effect). For strong magnets, the suppression of Andreev reflection may alter this picture, but also stray field effects become important, as is known from earlier work. We compare large-area samples with microstructured ones, and find blocklike switching in the latter. We show this not to be due to a switch between the P and AP states, but rather to dipolar coupling between domains which are forming in the two Py layers, making a stray-field scenario likely. We also present measurements of the depairing (critical) current $I_{d p}$ and show that a similar depression of superconductivity exists far below $T_{c}$ as is found around $T_{c}$.
\end{abstract}

DOI: 10.1103/PhysRevB.82.184523

PACS number(s): 74.45.+c, 74.78.Fk, 75.60.Ch

\section{INTRODUCTION}

In multilayers of superconducting (S) and ferromagnetic (F) materials, one of the phenomena which has received much attention in the last few years is the so-called superconducting spin-valve (SV) (or spin-switch) effect. First suggested by Buzdin et al. ${ }^{1,2}$ and by Tagirov, ${ }^{3}$ the device consists of an $\mathrm{F} / \mathrm{S} / \mathrm{F}$ sandwich where a superconducting spacer, with a thickness $d_{S}$ on the order of the superconducting coherence length $\xi_{S}$, separates two ferromagnetic layers with controllable magnetization directions. The calculations, performed in the weakly ferromagnetic limit (meaning equal spin subbands) showed that for such a device the superconducting transition temperature for parallel (P) alignment of the magnetization in both $\mathrm{F}$ layers, $T_{c}^{\mathrm{P}}$, is always lower than that for the antiparallel (AP) alignment $T_{c}^{\mathrm{AP}}$. Additionally, it should be possible to tune the device parameters (in particular, the $\mathrm{F}$ layer thicknesses $d_{F}$ ) such that superconductivity only appears in the AP alignment, leading to a controllable supercurrent through a small field manipulation. Experiments on macroscopic-sized spin-valve systems using weakly ferromagnetic $\mathrm{CuNi}$ appeared to follow the theoretical prediction, ${ }^{4,5}$ although the difference in $T_{c}$ between the $\mathrm{P}$ and the AP alignment was only a few millikelvin, much smaller than the calculations indicated, and reentrance was not found. Recent measurements on $\mathrm{S} / \mathrm{F}$ bilayers made of $\mathrm{Nb} /$ $\mathrm{CuNi}$ showed that a $T_{c}$ lowering of a few millikelvin is also found when the ferromagnet is in a domain state. ${ }^{6}$ This is basically due to the fact that Cooper pairs break less easily when they sample an inhomogeneous exchange field, which can be considered a lateral variant of the spin-valve effect. ${ }^{7}$

For spin-valve systems with strong ferromagnets, the situation is more complicated. In a number of reports, with magnets such as $\mathrm{Ni}$ (Ref. 8) or $\mathrm{Ni}_{80} \mathrm{Fe}_{20}$ (permalloy, Py), ${ }^{9,10}$ standard spin-valve behavior $T_{c}^{\mathrm{AP}}>T_{c}^{\mathrm{P}}$ was observed, with the difference between $T_{c}^{\mathrm{AP}}$ and $T_{c}^{\mathrm{P}}$ going up to $40 \mathrm{mK}$ at low temperatures. In other experiments with $\mathrm{Py},{ }^{11,12} \mathrm{Co}$, and $\mathrm{Fe}$ combined $^{13}$ or Co, ${ }^{14}$ "inverse" behavior $T_{c}^{\mathrm{AP}}<T_{c}^{\mathrm{P}}$ was found. This was explained as either due to enhanced reflection of spin-polarized quasiparticles (a mechanism not present in the calculations with equal spin subbands), ${ }^{11}$ or to the effects of stray fields from domain walls. ${ }^{10,12-14}$ The difference between the observations of the standard and the inverse effects appears to be that those experiments finding standard behavior make use of an antiferromagnetic pinning layer in order to have one layer with fixed, and one layer with a freely rotating magnetization, while the reports on the inverse effect use a difference of $d_{F}$ or different materials for both layers in order to create a difference in coercive fields and thereby a field range where the magnetizations are AP. The pinning layer suppresses domain formation, and, in particular, the work of Zhu et al. showed that if, in samples with a pinning layer, the free layer is brought in a domain state, the standard spin-valve effect is lost. ${ }^{10}$ Focusing now on samples without pinning layer, almost all investigations were performed on (millimeter) large samples, where large amount of domains were present during the transition from the $\mathrm{P}$ to the AP state. In the work of Rusanov et al., samples were structured down to micrometer size, and the switching of the magnetization was instantaneous, giving blocklike variations in the resistance, and suggesting that a clear P to AP switch was obtained. ${ }^{11}$ In such a case, it can still be argued that for strongly spin-polarized ferromagnets the $\mathrm{P}$ alignment may yield an enhanced $T_{c}$; in particular, in the limit of $100 \%$ spin polarization, the $\mathrm{P}$ alignment confines quasiparticles in the $\mathrm{S}$ layer because Andreev reflections are not possible while they still can leave the superconductor (one electron to each bank, in a crossed process) in the AP alignment.

In this work we examine the question whether also in micron-sized samples the inverse spin-valve effect is related to stray fields or due to the spin polarization. We present a series of measurements on $\mathrm{Nb} / \mathrm{Py}$ bilayers and spin-valve devices, both with large sizes and microsized, where we compare anisotropic magnetoresistance (AMR) effect in the normal state with the magnetoresistance measurements in the superconducting state. We find that, according to the AMR, domains also exist in micron-sized samples, and that the blocklike switching is presumably due to dipolar coupling between the domains in the two Py layers, acting as one. 


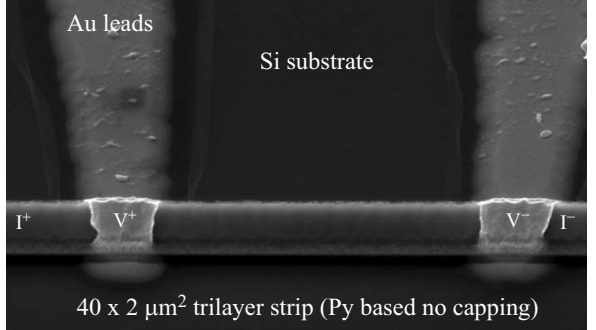

FIG. 1. SEM image of a four-probe $40 \times 2 \mu \mathrm{m}^{2}$ spin-valve device. The distance between the voltage probes $\left(V^{+}\right.$and $\left.V^{-}\right)$is $10 \mu \mathrm{m}$ and the full length of the spin-valve wire is $40 \mu \mathrm{m}$. Similar $\mathrm{Au}$ contacting leads for the current probes are at the end points of the wire (not shown).

This makes the stray field scenario likely. We also present measurements of the depairing (critical) current $I_{d p}$ far below $T_{c}$ and show that a similar depression of superconductivity exists in that temperature regime as found around $T_{c}$.

\section{EXPERIMENTAL DETAILS}

$\mathrm{Nb} / \mathrm{Py}$ layers were grown on $\mathrm{Si}(100)$ substrates by dc magnetron sputtering in a ultrahigh-vacuum chamber with a background pressure of $10^{-9}$ mbar and an Ar pressure of $4 \mu$ bar for the $\mathrm{Nb}$ and $2.5 \mu$ bar for the $\mathrm{Py}$, with $\mathrm{Nb}$ as bottom layer. The substrate holders were equipped with a small magnet to determine the direction of easy axis for the Py layers and enhance the fast switching properties. The $\mathrm{Nb}$ layer thickness was kept at $50 \mathrm{~nm}$ for all samples while for the Py layers thickness we used 20 and $50 \mathrm{~nm}$. For our Py/ $\mathrm{Nb} / \mathrm{Py}$ spin-valve devices the bottom Py layer is $50 \mathrm{~nm}$ thick and the top Py layer is $20 \mathrm{~nm}$ thick. All our devices have an additional $2 \mathrm{~nm} \mathrm{Nb}$ capping layer added on top to prevent oxidation of the top Py layer. We show in the Appendix that not adding such protective layer leads to an exchange-biased Py layer, likely due to antiferromagnetic iron-oxide regions. The Py has a degree of polarization close to $45 \%$ and a Curie temperature around $900 \mathrm{~K}$. The thickness of the layers is far larger than the length $\xi_{F}$ over which superconducting correlations are assumed to penetrate, which is around $1.5 \mathrm{~nm} .{ }^{15}$ The purity of the $\mathrm{Nb}$ target is $99.95 \%$ which yields a $T_{c}$ of 9.1-9.2 K for thick films. At the thickness of $50 \mathrm{~nm}, T_{c}$ of a single film is around $8.9 \mathrm{~K}$, and from critical field measurements preformed earlier on similar samples we extract a zero-temperature Ginzburg-Landau (GL) coherence length $\xi_{\mathrm{GL}}(0) \approx 13 \mathrm{~nm}$. This corresponds to a normal-state elastic mean-free path $\ell_{N} \approx 5.5 \mathrm{~nm}^{11}$

Standard electron-beam lithography was used to pattern both the microsized strips, with a length of $40 \mu \mathrm{m}$ and a width of 1 to $4 \mu \mathrm{m}$, and the large-scale devices (2000 $\times 200 \mu \mathrm{m}^{2}$ ), where the direction of the strips are aligned with magnetic easy axis of the Py layer. These strips were etched down using Ar ion etching at a Ar pressure of $3 \mu \mathrm{bar}$ with a background pressure of $10^{-6}$ mbar. Au contacts were sputtered in a second deposition step using a lift-off resist mask technique. A few nanometer of Ti were sputter as adhesion layer for the $\mathrm{Au}$. The contact geometry is four probe, with $10 \mu \mathrm{m}$ spacing between the voltage probes. Figure 1

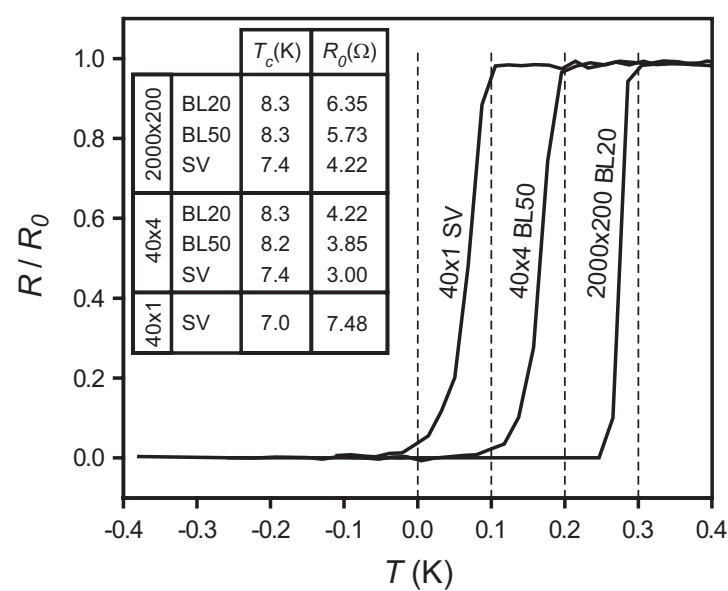

FIG. 2. Temperature variation in the resistance around the transition temperature $T_{c}$ for (from left to right) a $\mathrm{Py}(50) / \mathrm{Nb}(50) / \mathrm{Py}(20)$ spin valve (SV), a $\mathrm{Nb}(50) / \mathrm{Py}(50)$ bilayer (BL50) and a $\mathrm{Nb}(50)$ / $\mathrm{Py}(20)$ bilayer (BL20), with numbers representing the layer thickness in nanometer. Resistance is normalized to the low-temperature resistance $R_{0}$ and curves are shifted along temperature for clarity. Lateral dimensions of the devices are given in the graph in units of $\mu \mathrm{m}^{2}$. The table shows $T_{c}$ and $R_{0}$ of all devices presented in this report.

shows a scanning electron microscope (SEM) image of a $40 \times 2 \mu \mathrm{m}^{2}$ spin valve. This recipe is used for both bilayer and spin valve, both for microsized and macrosized devices although for the latter a spacing of $1000 \mu \mathrm{m}$ between the voltage probes was used. Typical transition curves (resistance $R$ versus temperature $T$ around $T_{c}$ ) for several of our devices are shown in Fig. 2, where for clarity curves are shifted along temperature. The typical width of the transition is $50-100 \mathrm{mK}$. Also shown in the figure are all $T_{C}$ 's and low temperature resistances $\left(R_{0}\right)$ for our devices. The choice for different layer thicknesses for the Py in the spin-valve device is to establish different coercive fields, making the device switchable from parallel to antiparallel. The coercive fields of the 20- and 50-nm-thick microsized Py strips are expected to be in the range from 0 to $20 \mathrm{mT}$ (Ref. 11) with a wider strip leading to a lower coercive field value (a large Py thin film of $20 \mathrm{~nm}$ thickness was measured to have a coercive field less than $0.5 \mathrm{mT}$ ). For our typical microsized strips (1-4 $\mu \mathrm{m}$ wide) we always found a difference of about $5 \mathrm{mT}$ between the two different thicknesses. However, as we will show later, the mutual influence of the dipole fields prevents clean and uncorrelated switching. The microsized elongated structures were chosen to promote single domain switching. They also have a sufficiently large cross-sectional resistance to perform critical current $\left(I_{c}\right)$ measurements. We performed AMR measurements just above $T_{c}$ to see if and when domains appear in our $\mathrm{Nb} / \mathrm{Py}$ bilayer systems, and compared this to the AMR signal of the (spin-valve) trilayers. The response of the superconductor was found by comparing these AMR measurements to the magnetoresistance measurements in the transition. All measurements were done in a standard ${ }^{4} \mathrm{He}$ cryostat with magnetic shielding to provide a low-noise environment. It is equipped with a superconducting coil to generate magnetic fields up to $1 \mathrm{~T}$. All field measurements 


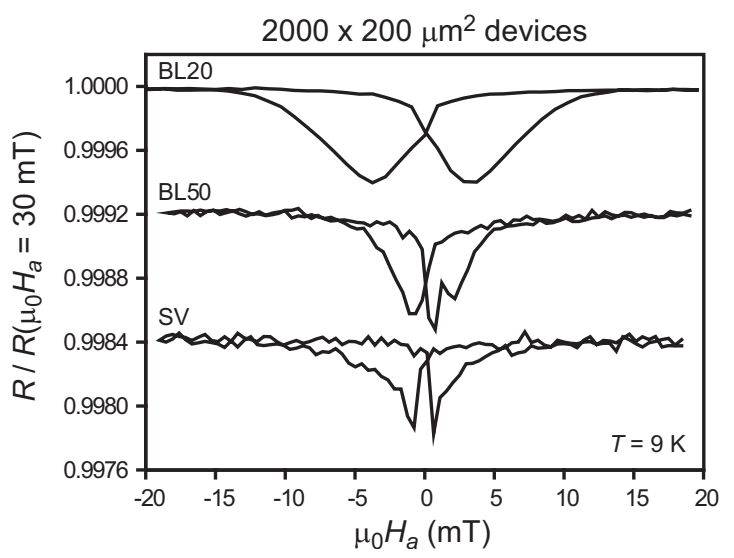

FIG. 3. Resistance normalized to the value at $30 \mathrm{mT}$ as function of the in-plane applied field $H_{a}$ on large-scale devices with lateral dimension $2000 \times 200 \mu \mathrm{m}^{2}$, all at temperature $T=9 \mathrm{~K}$. From top to bottom, a $\mathrm{Nb}(50) / \mathrm{Py}(20)$ bilayer (BL20), a $\mathrm{Nb}(50) / \mathrm{Py}(50)$ bilayer (BL50), and a $\mathrm{Py}(50) / \mathrm{Nb}(50) / \mathrm{Py}(20) \mathrm{SV}$, with numbers representing the layer thickness in nm. The BL50 and SV curves are shifted by, respectively, -0.0008 and -0.0016 .

were performed with the direction of the applied field along the long side of the strip, which implies field parallel to the measurement current. The critical current measurements were performed well below $T_{c}$, and probe the gap strength, enabling a comparison between $\mathrm{P}$ and AP below the transition area. A pulsed current method was used for these measurements, which is described in Ref. 16.

\section{RESULTS}

\section{A. $R(H)$ for $T>T_{c}$}

Figure 3 shows the result of $R(H)$ measurements on largescale $\mathrm{Nb} / \mathrm{Py}$ bilayers and a $\mathrm{Py} / \mathrm{Nb} / \mathrm{Py}$ spin valve, all with lateral dimension $2000 \times 200 \mu \mathrm{m}^{2}$ and at a temperature of 9 $\mathrm{K}$ ( $\mathrm{Nb}$ in normal state). All devices show the characteristic AMR dip, with a relative resistance change close to $0.06 \%$. The spin valve and bilayer with the thick Py layer $(50 \mathrm{~nm})$ show a very similar hysteretic curve with a coercive field value close to $1 \mathrm{mT}$; the bilayer with the thin Py layer $(20$ $\mathrm{nm}$ ) shows a much broader dip with a coercive field close to $3 \mathrm{mT}$. The same measurements but now on microstructured strips, all with lateral dimension $40 \times 4 \mu \mathrm{m}^{2}$, are shown in Fig. 4. For the spin valve, block-shaped hysteretic dips appear with switches near $\pm 1 \mathrm{mT}$ and $\pm 3 \mathrm{mT}$, and with (again) a relative resistance change of $0.06 \%$. For both the 50-nm-thick and 20-nm-thick bilayer we do not see any AMR dip coming out of the measurement noise, pointing toward a single domain type of switching. The noise level is similar for all three devices and about $0.01 \%$, which is $0.3-0.4 \mathrm{~m} \Omega$ in terms of absolute resistance value. It is significantly worse compared to the large-scale devices and suggests that contacts to the strip are of less quality.

The appearance of this (seemingly) two-step switch process in the microsized spin valve is very different from the large-scale spin valve. Yet, the size of the resistance change (about $24 \mathrm{~m} \Omega$ ) is similar, and the observed switching fields

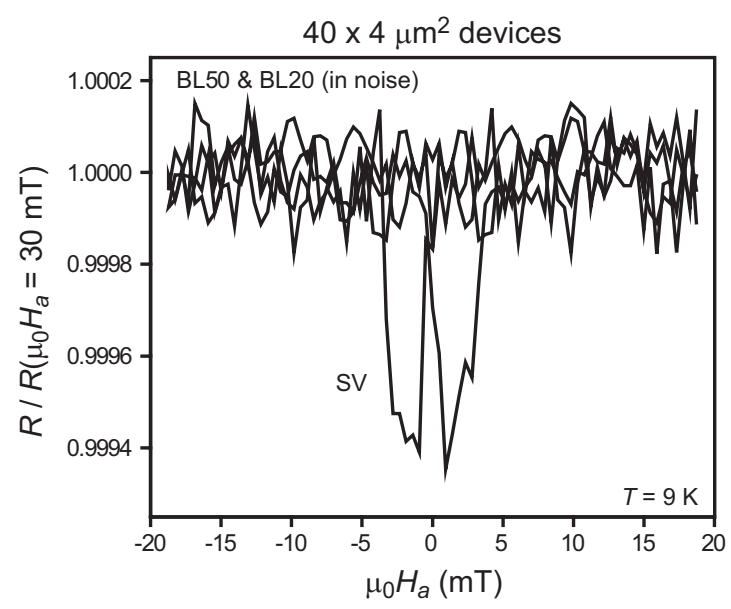

FIG. 4. Resistance normalized to the value at $30 \mathrm{mT}$ as a function of the in-plane applied field $H_{a}$ on microsized devices with lateral dimension $40 \times 4 \mu \mathrm{m}^{2}$, all with temperature $T=9 \mathrm{~K}$. The presented curve is from a $\mathrm{Py}(50) / \mathrm{Nb}(50) / \mathrm{Py}(20)$ spin valve while the results on the $\mathrm{Nb}(50) / \mathrm{Py}(20)$ and $\mathrm{Nb}(50) / \mathrm{Py}(50)$ bilayers (BL20 and BL50) are "flat" and within the noise of the spin valve, with numbers representing the layer thickness in nanometer.

of the blocks coincide with the coercive fields of the two different large-scale bilayers. To further explore this block type of switching we fabricated a more narrow bridge $(1 \mu \mathrm{m}$ wide) increasing the shape anisotropy energy, thus enhancing its importance in determining the possible domain states in the strips. Results are presented in Fig. 5 and show a series of $R(H)$ measurements at constant temperature $(T=9 \mathrm{~K})$ above

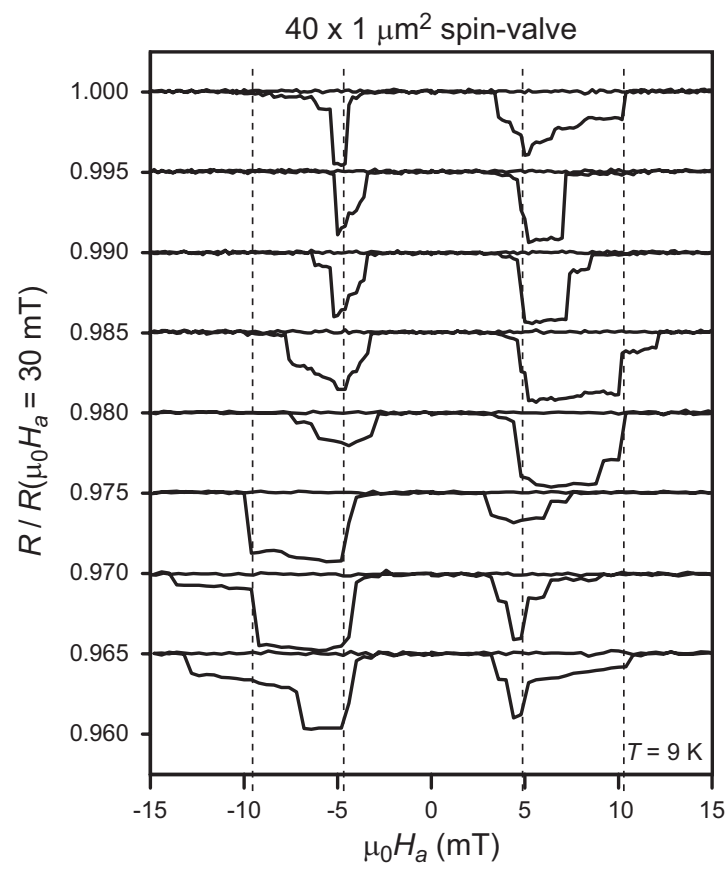

FIG. 5. Resistance normalized to the value at $30 \mathrm{mT}$ as function of the in-plane applied field $H_{a}$ on a $40 \times 1 \mu \mathrm{m}^{2} \mathrm{Py}(50) / \mathrm{Nb}(50) /$ $\mathrm{Py}(20)$ spin valve, with numbers representing the layer thickness in nanometer. All curves have a different magnetic history and are repeatedly shifted by -0.005 . For all measurements the temperature was $9 \mathrm{~K}$. 
$T_{c}$, all with slightly different saturation histories. Although no two curves are identical, there seem to be only a limited number of values for the applied field where a jump in resistance is seen, and the size of those jumps take only few different values. The range over which hysteresis is found goes from $\pm(4-14) \mathrm{mT}$, which is significantly higher than in the other devices. Also the size of the resistance change is about ten times higher than in our wider devices, implying that more perpendicular domains (magnetization perpendicular to the current direction) are sampled.

The (almost) single block-type switching indicates that the $\mathrm{F}$ layers become magnetically coupled in the spin-valve device. This coupling appears to be such that a switching in the thinner layer (highest coercive field) is triggered by the switching of the thicker layer (lowest coercive field). In the $40 \times 4 \mu \mathrm{m}^{2}$ bilayer strips, the switching is not accompanied by domain formation but rather makes a fast single switch, most likely due to the enhanced shape anisotropy which favors single domain switching. In a spin-valve device of the same lateral dimension the F layers become coupled, resulting in a block-type switch process for the spin valve and the formation of domains. The measurements on the 40 $\times 1 \mu \mathrm{m}^{2}$ spin valve show that a variety of possible domain states exists, which can be entered during the switching. In wider strips we have never observed such behavior.

\section{B. $R(H)$ for $T<T_{c}$}

A similar set of measurements is performed inside the transition, with $\mathrm{Nb}$ superconducting. The $R(H)$ are all taken at temperatures near the low end of the transition curve to improve temperature stability. The measured signal now comes predominantly from the superconductor which is shorting the ferromagnetic layers by percolation paths. Furthermore, AMR features are no longer visible due to the (relative) high $\partial R / \partial T$ in the transition. Our typical $100 \mathrm{mK}$ transition width, combined with a $10^{-4}$ relative resistance change corresponds to a temperature change of $0.01 \mathrm{mK}$, which is below our measurement control of about $0.3 \mathrm{mK}$. Figure 6 shows the result of $R(H)$ measurements on the large-scale devices $\left(2000 \times 200 \mu \mathrm{m}^{2}\right)$. While the bilayer with the 50-nm-thick Py layer does not show any hysteric feature, the bilayer with the 20-nm-thick Py layer shows clear hysteric peaks. Their location is around $\pm 8 \mathrm{mT}$, which is significantly higher than the corresponding AMR dips in the same device at $\pm 3 \mathrm{mT}$. Also the spin valve shows such hysteretic peaks but located at lower fields around $\pm 1.5 \mathrm{mT}$, only slightly higher than the corresponding AMR dips in the same device. For the microsized devices $\left(40 \times 4 \mu \mathrm{m}^{2}\right)$ the same trend is observed (see Fig. 7) with again no hysteretic feature in the bilayer with the 50-nm-thick Py layer while the bilayer with the 20-nm-thick Py layer and the spin valve both do show hysteretic peaks. In case of the bilayer, the peaks are located at $\pm 4 \mathrm{mT}$ (a lower value compared to the largescale devices). For the spin valve the peaks are now really block shaped, with switching fields at $\pm 1 \mathrm{mT}$ and $\pm 4 \mathrm{mT}$. In Fig. 8 we present the $R(H)$ measurements on the smaller spin valve $\left(40 \times 1 \mu \mathrm{m}^{2}\right)$, and make a direct comparison with the obtained $T>T_{c}$ results (of the same sample, see Fig. 5).

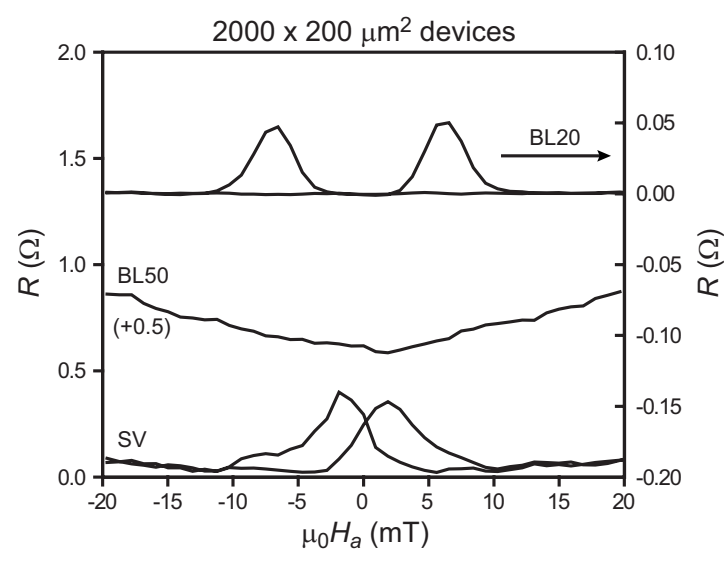

FIG. 6. Resistance as a function of the in-plane applied field $H_{a}$ on large-scale devices with lateral dimension $2000 \times 200 \mu \mathrm{m}^{2}$, at temperatures on the low end of the transition curve ( $\mathrm{Nb}$ in superconducting state). From top to bottom, a $\mathrm{Nb}(50) / \mathrm{Py}(20)$ bilayer (BL20), a $\mathrm{Nb}(50) / \mathrm{Py}(50)$ bilayer (BL50), and a $\mathrm{Py}(50) / \mathrm{Nb}(50) /$ $\mathrm{Py}(20) \mathrm{SV}$, with numbers representing the layer thickness in $\mathrm{nm}$. The BL50 curve is shifted by +0.5 and the BL20 curve is on the right-hand scale.

Block-shaped peaks are observed (where all measurements have different magnetization history) with switches at \pm 5 and $\pm 10 \mathrm{mT}$. Furthermore, there is a striking resemblance between the observed blocks for $T>T_{c}$ (dips) and $T<T_{c}$ (peaks).

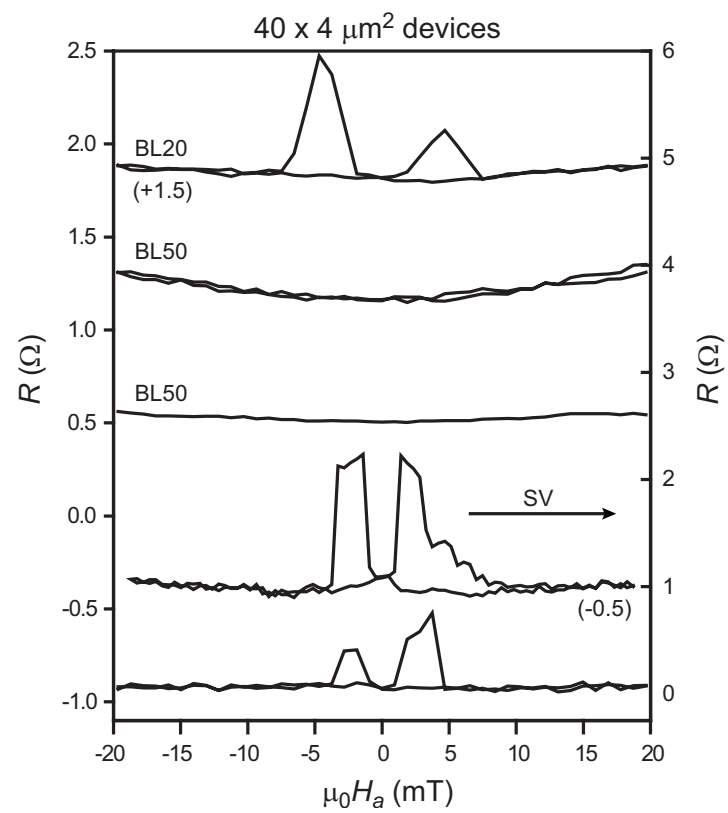

FIG. 7. Resistance as a function of the in-plane applied field $H_{a}$ on microsized devices with lateral dimension $40 \times 4 \mu \mathrm{m}^{2}$, at temperatures on the low end of the transition curve $(\mathrm{Nb}$ in superconducting state). From top to bottom, a $\mathrm{Nb}(50) / \mathrm{Py}(20)$ bilayer (BL20), $2 \times$ a $\mathrm{Nb}(50) / \mathrm{Py}(50)$ bilayer (BL50), and $2 \times$ a $\mathrm{Py}(50) / \mathrm{Nb}(50) /$ $\mathrm{Py}(20) \mathrm{SV}$, with numbers representing the layer thickness in nanometer. Two curves are shifted (by -0.5 and +1.5 ) as indicated. The SV curves are on the right-hand scale. 




FIG. 8. Comparison of the resistance as a function of the inplane applied field $H_{a}$ on a $40 \times 1 \mu \mathrm{m}^{2} \mathrm{Py}(50) / \mathrm{Nb}(50) / \mathrm{Py}(20)$ spin valve (numbers representing the layer thickness in nanometer) between temperatures on the low end of the transition curve $(\mathrm{Nb}$ in superconducting state) and above the transition temperature $T_{c}(\mathrm{Nb}$ in normal state). All curves have a different magnetic history. Lefthand scale: results for $T<T_{c}$ with curves repeatedly shifted by +2.5 as indicated. Right-hand scale: results for $T>T_{c}$ taken from Fig. 5.

\section{C. $I_{c}(H)$ for $T$ well below $T_{c}$}

The measurements so far all focus on temperatures closely around the transition $\left(T \sim T_{c}\right)$. To study the working of the spin valve well below $T_{c}$ we conducted a series of critical current measurements as function of applied field, $I_{c}\left(H_{a}\right)$ by measuring the current $I$-voltage $V$ characteristic. We used $3 \mathrm{~ms}$ current pulses, with an interval of several seconds and increasing in amplitude until the critical current is reached and the superconductor is driven in the normal state. The sample is initially cooled down in zero-field condition and the first measurement at a fixed temperature always starts in zero applied field. The obtained $I-V$ curves all showed a sharp jump from almost zero voltage to the normal state, which indicates that we are measuring a depairing current rather than the onset to vortex flow. This we (consistently) found before on S films and S/F bilayers. ${ }^{6,16,17}$ The measurement is thus directly sensitive to the amplitude of the superconducting gap, which limits the critical current. Additionally, the value of $I_{c}$ is well defined due to the sharp transition. The results for the $40 \times 4 \mu \mathrm{m}^{2} \mathrm{Nb} / \mathrm{Py}$ spin valve are shown in Fig. 9, where $I_{c}(H)$ curves are presented at four temperatures well below $T_{c}$, which, in terms of the reduced temperature $t=T / T_{c}$ go down to $t=0.5$. All data show a blocklike dip for the increasing applied field (coming from negative saturation) with switching fields around 0.5 and 3.5 $\mathrm{mT}$, after which the curve becomes constant. The observed switching fields of the blocks do not show a temperature dependence but do show a diminishing effect for decreasing temperature. The uncertainty in the determination of $I_{c}$ is about the step size for the increase in current $(1 \mu \mathrm{A})$. We interpret the lowering of $I_{c}$ as a suppression of the supercon-

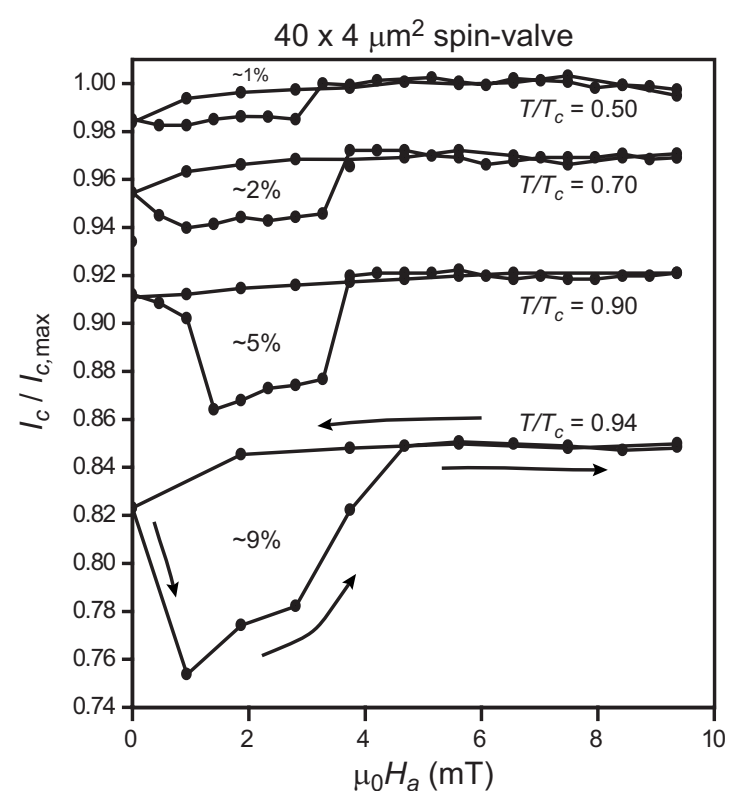

FIG. 9. Critical current measurements $I_{c}$, normalized to the maximum observed value $I_{c \text {, max }}$ for each temperature, as function of the in-plane applied field $H_{a}$ on a $40 \times 4 \mu \mathrm{m}^{2} \mathrm{Py}(50) / \mathrm{Nb}(50) /$ $\mathrm{Py}(20)$ spin valve. The curves represent different reduced temperatures $T / T_{c}$ and are shifted for clarity by $-0.03,-0.08$ and -0.15 . The indicated percentages are the relative sizes of the dips. Arrows denote the direction of the field sweep.

ducting gap. Because the gap increases in strength for lower temperature, it is not strange to see a diminishing effect of the percentage change. The switching fields coincide with the values found in the transport measurements close to $T_{c}$ (see Figs. 4 and 7). Figure 10 shows the $t=0.94$ curve for up to higher field values, and the standard decrease in $I_{c}$ due to applied field becomes visible. Additionally, in the inset the actual $I-V$ measurement at the highest used field (125 mT).

\section{DISCUSSION AND CONCLUSIONS}

Summarizing the data, we can come to the following conclusions. For temperatures above the transition ( $\mathrm{Nb}$ in nor-



FIG. 10. High-field behavior of the $T / T_{c}=0.94$ critical current measurement as presented in Fig. 9. The inset shows the current voltage measurement of the curve for $\mu_{0} H_{a}=125 \mathrm{mT}$. 
mal state) we find that the resistance changes are dominated by the AMR effect of the Py layers, with a relative resistance change of order $10^{-4}$. In large-scale devices, where domain formation is not limited by the size of the sample, the observed resistance dips in the bilayers with 20-nm- and 50nm-thick Py appear at different fields. For the spin-valve structure we then expect to see all four resistance dips in the AMR. However, we observe a behavior very similar to the bilayer with 50-nm-thick Py, already giving an indication for coupling effects between the two F layers.

Going to the microscopic regime, we no longer observe any dips in the AMR signal of the bilayers, pointing to a fast single domain type of switching. However, in the spin valve we do observe AMR resistance changes. A two-stepped switching has appeared by going from large scale to microsized spin valves, most strongly pronounced in the thinnest (1 $\mu \mathrm{m}$ wide) spin-valve structure. This is a strong indication that the single domain switching in the bilayers is replaced by domain formation, including magnetic coupling between the two Py layers.

For temperatures below the transition ( $\mathrm{Nb}$ superconducting) we observe peaks in the resistance now originating from changes in the superconducting gap, with a relative resistance change of order $10^{-1}$. Especially in the $1-\mu \mathrm{m}$-wide spin valve, these peaks are mirror images of the dips in the corresponding AMR signals. This implies that the superconductor does not influence/change the switching behavior of the spin valve; and that the suppression of the superconductor is a direct effect of the presence of the stray fields from the coupled domains. Such coupling between the F layers was found in experiments using antiferromagnetic pinning layers.

In the bilayers we only observe these peaks in the devices with 20-nm-thick Py but not in the devices with 50-nm-thick Py. This we attribute to different types of domain walls. It is known that for very thin Py layers the domain wall becomes of the Néel type while for thicker Py layers it is Bloch type. ${ }^{18}$ The crossover between the two is around a Py thickness of $35 \mathrm{~nm}$, implying Néel walls in our 20-nm-thick Py bilayer and Bloch walls in our 50-nm-thick Py bilayers. Calculations on stray fields generated by domain walls shows a significantly higher magnitude for Néel walls than for Bloch walls, ${ }^{19}$ which we believe is the source of the observed difference in our bilayers.

A special remark concerns the point that no traces are found of an enhancement of the superconductivity by domain averaging from Cooper pairs, as found before both in $\mathrm{CuNi}$ - and Py-based devices, 6,7 and also more recently in a different set of Py-based devices. ${ }^{20}$ In particular, for the large-area bilayer sample BL20, enhanced superconductivity in the domain state might have been expected. This is most probably due to the fact that the switching behavior and domain formation in Py are very sensitive to the relative orientations of easy axis and applied field (see also Ref. 21). Although the samples were grown in a small bias field, no further special alignment was performed in the present experiments, and stray fields now apparently win from domain averaging. Note, incidentally, that even stray fields can lead to $T_{c}$ enhancement in $\mathrm{F} / \mathrm{S} / \mathrm{F}$ structures but that needs carefully designed samples. ${ }^{22}$

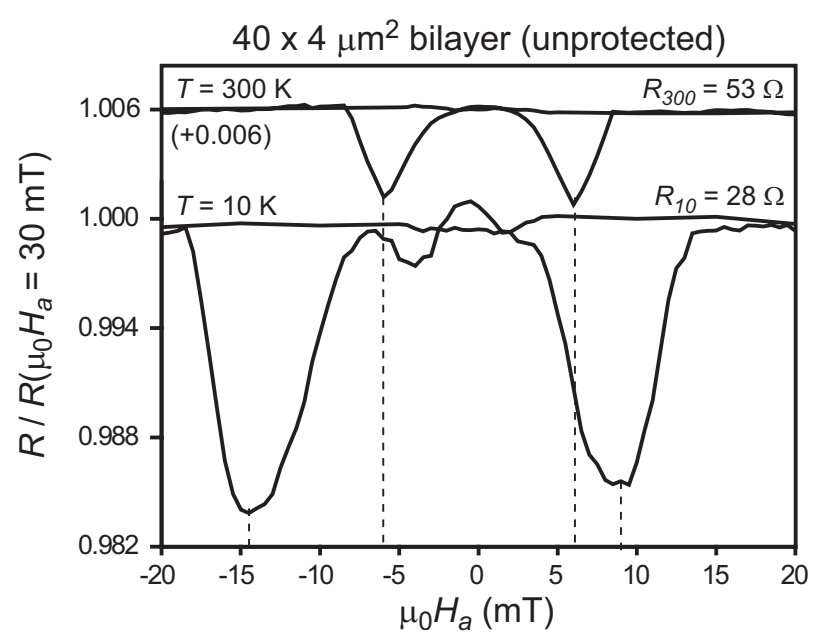

FIG. 11. Resistance normalized to the value at $30 \mathrm{mT}$ as a function of the in-plane applied field $H_{a}$ on a $40 \times 4 \mu \mathrm{m}^{2} \mathrm{Nb} / \mathrm{Py}$ bilayer, for $T=300 \mathrm{~K}$ and $T=10 \mathrm{~K}$. The respective resistances are indicated by $R_{300}$ and $R_{10}$. Both the Nb layer and Py layer are $20 \mathrm{~nm}$ thick.

By going to lower temperatures and measuring the critical current, which is a direct measure for the superconducting gap strength, we observe that the suppression of superconductivity is still present. The fields at which the suppression occurs overlaps with the peaks in the magnetotransport measurements and do not change with temperature. This indicates that also a well-developed gap is not changing the switching of the Py layers, and likely the spin valve is still dominated by the stray fields. In our $I-V$ measurements we do not see traces of a vortex flow while the stray fields connecting the two F layers should result in vortices. However, since the domain state seems to be unaffected by the gap, we believe it strong enough to keep any vortices in place. Effectively, all vortices generated by the stray fields are strongly pinned by the (rigid) domain state itself.

\section{ACKNOWLEDGMENTS}

We acknowledge C. Bell and M. Hesselbert for discussions and technical support. This work is part of the research program of the Foundation for Fundamental Research on Matter (FOM), which is part of the Netherlands Organization for Scientific Research (NWO).

\section{APPENDIX: EXCHANGE BIASED Py}

All presented data so far were on devices where the top Py layer is covered by a thin $\mathrm{Nb}$ layer to protect it from oxidizing. Magnetoresistance measurements on a 40 $\times 2 \mu \mathrm{m}^{2}$ bilayer strip without such capping layer is presented in Fig. 11, where $R(H)$ at room temperature $(T$ $=300 \mathrm{~K})$ is compared with low temperature $(T=10 \mathrm{~K})$. At room temperature the AMR signal contains the typical dips. They are symmetrical around zero field, with a coercive field of $\pm 6 \mathrm{mT}$, and with a relative resistance change of 0.05 . At 
low temperature the dips have become broader, the coercive fields have become larger but the curve is no longer symmetric around zero field. The coercive fields are now at -14 and $9 \mathrm{mT}$, which indicates an exchange bias of $2.5 \mathrm{mT}$ (such that unbiased the coercive fields would be symmetric again at $\pm 11.5 \mathrm{mT}$ ). We expect this exchange bias to be the result of the formation of $\mathrm{Fe}_{2} \mathrm{O}_{3}$ at the top of the Py layer, which becomes antiferromagnetic below $250 \mathrm{~K}$.
${ }^{1}$ A. I. Buzdin, A. V. Vedyayev, and N. V. Ryzhanova, Europhys. Lett. 48, 686 (1999).

${ }^{2}$ A. I. Buzdin, Rev. Mod. Phys. 77, 935 (2005).

${ }^{3}$ L. R. Tagirov, Phys. Rev. Lett. 83, 2058 (1999).

${ }^{4}$ J. Y. Gu, C.-Y. You, J. S. Jiang, J. Pearson, Y. B. Bazaliy, and S. D. Bader, Phys. Rev. Lett. 89, 267001 (2002).

${ }^{5}$ A. Potenza and C. H. Marrows, Phys. Rev. B 71, 180503(R) (2005).

${ }^{6}$ M. Flokstra and J. Aarts, Phys. Rev. B 80, 144513 (2009).

${ }^{7}$ A. Y. Rusanov, M. Hesselberth, J. Aarts, and A. I. Buzdin, Phys. Rev. Lett. 93, 057002 (2004).

${ }^{8}$ I. C. Moraru, W. P. Pratt, Jr., and N. O. Birge, Phys. Rev. Lett. 96, 037004 (2006).

${ }^{9}$ I. C. Moraru, W. P. Pratt, and N. O. Birge, Phys. Rev. B 74, 220507(R) (2006).

${ }^{10}$ J. Zhu, X. Cheng, C. Boone, and I. N. Krivorotov, Phys. Rev. Lett. 103, 027004 (2009).

${ }^{11}$ A. Y. Rusanov, S. Habraken, and J. Aarts, Phys. Rev. B 73, 060505(R) (2006).

${ }^{12}$ D. Stamopoulos, E. Manios, and M. Pissas, Phys. Rev. B 75,
184504 (2007).

${ }^{13}$ R. Steiner and P. Ziemann, Phys. Rev. B 74, 094504 (2006).

${ }^{14}$ G. Carapella, F. Russo, and G. Costabile, Phys. Rev. B 78, 104529 (2008).

${ }^{15}$ J. W. A. Robinson, S. Piano, G. Burnell, C. Bell, and M. G. Blamire, Phys. Rev. Lett. 97, 177003 (2006).

${ }^{16}$ A. Y. Rusanov, M. B. S. Hesselberth, and J. Aarts, Phys. Rev. B 70, 024510 (2004).

${ }^{17}$ C. Cirillo, A. Rusanov, C. Bell, and J. Aarts, Phys. Rev. B 75, 174510 (2007).

${ }^{18}$ T. Trunk, M. Redjdal, A. K'akay, M. F. Ruane, and F. B. Humphrey, J. Appl. Phys. 89, 7606 (2001).

${ }^{19}$ M. Redjdal, J. H. Giusti, M. F. Ruane, and F. B. Humphrey, IEEE Trans. Magn. 39, 2684 (2003).

${ }^{20}$ A. Y. Rusanov, T. E. Golikova, and S. V. Egorov, JETP Lett. 87, 175 (2008).

${ }^{21}$ T.-J. Hwang and D. H. Kim, J. Korean Phys. Soc. 48, 1133 (2006).

${ }^{22}$ W. Gillijns, A. Y. Aladyshkin, M. Lange, M. J. Van Bael, and V. V. Moshchalkov, Phys. Rev. Lett. 95, 227003 (2005). 